\title{
Echo Trains in Pulsed Electron Spin Resonance of a Strongly Coupled Spin Ensemble
}

\author{
Stefan Weichselbaumer, ${ }^{1,2}$ Matthias Zens, ${ }^{3,4}$ Christoph W. Zollitsch, ${ }^{1,2, *}$ Martin S. Brandt, ${ }^{5,2,6}$ \\ Stefan Rotter $\oplus^{3}$ Rudolf Gross, ${ }^{1,2,6}$ and Hans Huebl $\oplus^{1,2,6, \dagger}$ \\ ${ }^{1}$ Walther-Meißner-Institut, Bayerische Akademie der Wissenschaften, 85748 Garching, Germany \\ ${ }^{2}$ Physik-Department, Technische Universität München, 85748 Garching, Germany \\ ${ }^{3}$ Institute for Theoretical Physics, TU Wien, Wiedner Hauptstraße 8-10/136, 1040 Vienna, Austria \\ ${ }^{4}$ ITAMP, Harvard-Smithsonian Center for Astrophysics, Cambridge, Massachusetts 02138, USA \\ ${ }^{5}$ Walter Schottky Institut, Technische Universität München, 85748 Garching, Germany \\ ${ }^{6}$ Munich Center for Quantum Science and Technology (MCQST), Schellingstraße 4, 80799 München, Germany
}

(Received 26 September 2018; revised 15 July 2020; accepted 5 August 2020; published 22 September 2020)

\begin{abstract}
We report on a novel dynamical phenomenon in electron spin resonance experiments of phosphorus donors. When strongly coupling the paramagnetic ensemble to a superconducting lumped element resonator, the coherent exchange between these two subsystems leads to a train of periodic, self-stimulated echoes after a conventional Hahn echo pulse sequence. The presence of these multiecho signatures is explained using a simple model based on spins rotating on the Bloch sphere, backed up by numerical calculations using the inhomogeneous Tavis-Cummings Hamiltonian.
\end{abstract}

DOI: 10.1103/PhysRevLett.125.137701

Pulsed electron spin resonance (ESR) is an essential spectroscopy technique used in many fields of science, e.g., for the study of the structure and dynamics of molecular systems [1,2], and for material science [3], as well as for quantum sensing and information applications [4-6]. To implement this technique, a vast repertoire of sophisticated pulse sequences exists [7], each of them optimized to investigate particular spin properties. Nevertheless, the majority of the sequences is based on a Hahn echo [8], as schematically shown in Fig. 1(a).

A newly emerging area for ESR experiments is the processing of quantum information. Using superconducting microwave resonators, the so-called strong coupling regime has recently been demonstrated [9-14]. Here, the coherent exchange of information between the microwave resonator and the spin ensemble exceeds the individual decay rates of the two subsystems, which is a requirement for applications involving the storage and conversion of quantum information [9,15-17]. Apart from its importance for quantum technology, a strong coupling rate also enhances the sensitivity in ESR applications [18,19], going beyond classical ESR models [7,20]. First seminal experiments in the presence of strong spin-photon coupling revealed a plethora of new physical effects [13,21-23]. A fascinating question that remains unresolved, however, is what happens when the Hahn echo is transferred to the context of a strongly coupled spin ensemble.

To explore this question experimentally, we work with a superconducting microwave resonator strongly coupled to a paramagnetic spin ensemble. Specifically, we compare pulsed ESR measurements of a strongly coupled spin ensemble based on isolated phosphorus donors in a ${ }^{28} \mathrm{Si}$ host matrix with a weakly coupled ensemble of $\mathrm{P}_{2}$ dimers also present in the sample. In the weak coupling case, as in a conventional ESR experiment, we observe a single Hahn echo in terms of a photon pulse that is emitted into the resonator at $2 \tau$ when the spins refocus, where $\tau$ is the interpulse delay. In stark contrast, when applying the same Hahn echo sequence in the strong coupling regime, we observe a periodic sequence of spin echo signatures spaced by $\tau$. Although this phenomenon has been reported for up to two echoes earlier [24], it was not set in context with the strong coupling regime and a thorough understanding of the underlying mechanism is missing. Here we show that the formation of self-stimulated echoes is a robust phenomenon and can be well understood based on the inhomogeneous Tavis-Cummings model.

Our experimental scheme is shown in Figs. 1(b)-1(d) and consists of a planar superconducting lumped element resonator (LER), which is patterned into a $150 \mathrm{~nm}$ thin $\mathrm{Nb}$ film on an intrinsic nat ${ }^{\mathrm{Si}}$ substrate (see Supplemental Material [25]). The LER is located next to a microwave feedline, allowing us to probe the complex microwave transmission of the device. A $20 \mu \mathrm{m}$ thin slab of [100] oriented ${ }^{28} \mathrm{Si}: \mathrm{P}$ is mounted onto the LER [see Fig. 1(d)] and investigated at a temperature of $T=50 \mathrm{mK}$. A static magnetic field $B_{0}$ is applied parallel to the $\mathrm{Nb}$ film to avoid degradation of its superconducting properties. We perform continuous-wave (cw) ESR by measuring the microwave transmission $\left|S_{21}\right|^{2}$ of the chip using a vector network analyzer. For pulsed ESR experiments, we digitize the echo signal using a heterodyne down-conversion scheme [25].

Continuous-wave ESR spectroscopy.-We first perform cw ESR spectroscopy to precharacterize the sample. 
(a)



(1) $z \quad$ (2)

(3)

(4)

(5)

(6)
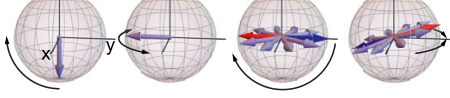

(b)
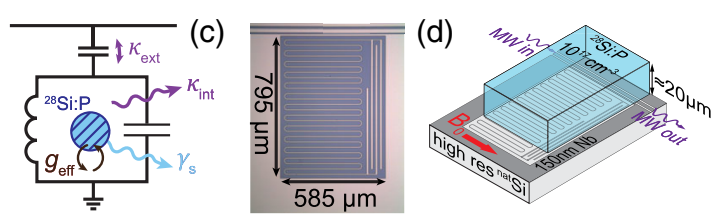

(e)

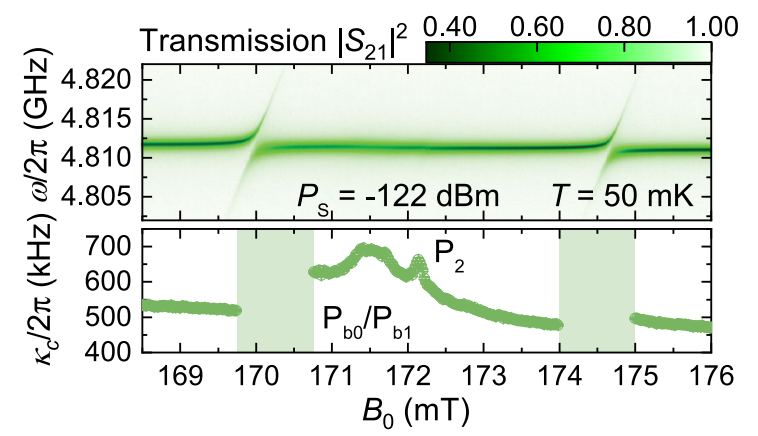

FIG. 1. (a) Schematic of the Hahn echo sequence and the associated states in the Bloch sphere (exterior black arrows indicate the ensuing spin dynamics). A $\pi / 2$ pulse is applied between (1) and (2) and an imperfect $\pi$ pulse between (3) and (4), leading to the first (conventional) Hahn echo between (5) and (6). For the subsequent pulse train observed in the strong coupling limit, the spin packets indicated by blue and red arrows are crucial, which lie in opposite $S_{y}$ directions when the first $\pi$ pulse arrives at (3) (see text). In (5)-(7) only these two spin packets are shown for better visibility. (b) Circuit diagram and (c) microscope image of the LER. (d) Schematic of the $20 \mu \mathrm{m}$ thin ${ }^{28} \mathrm{Si}$ :P sample mounted on top of the LER, also indicating the in-plane magnetic field direction $B_{0}$. (e) Normalized transmission $\left|S_{21}\right|^{2}$ as a function of frequency and magnetic field. Two avoided crossings are visible, indicating strong coupling between the hyperfine-split transitions of the phosphorus donors and the resonator. (f) Extracted linewidth $\kappa / 2 \pi$ (HWHM) as a function of magnetic field. Two additional spectroscopic features are observed, which are attributed to dangling bond defects $\mathrm{P}_{b 0} / \mathrm{P}_{b 1}$ and $\mathrm{P}_{2}$ dimers, respectively (see text).

Figure 1(e) shows the normalized microwave transmission $\left|S_{21}\right|^{2}$ for an incident power on the sample of $P_{S}=$ $-122 \mathrm{dBm}$. At $B_{0}=168.5 \mathrm{mT}$, we observe a bare resonator frequency of $\omega_{c} / 2 \pi=4.8116 \mathrm{GHz}$. Using a robust circle-fitting algorithm [58], we determine a half width at half maximum (HWHM) linewidth of $\kappa_{c} / 2 \pi=534.85 \mathrm{kHz}$, corresponding to a total quality factor of $Q=\omega_{c} / 2 \kappa_{c}=$ 4498. The coupling rate of the resonator to the feedline is $\kappa_{\text {ext }} / 2 \pi=304.15 \mathrm{kHz}$. Similarly, we extract the spin relaxation rate using a Lorentzian fit along the field axis far detuned from the resonator and find $\gamma_{s} / 2 \pi=279.03 \mathrm{kHz}$. We observe two distinct avoided crossings at $B_{0}=170.1$ and $B_{0}=174.3 \mathrm{mT}$, which are associated with the two hyperfine-split lines of phosphorus donors in silicon. The presence of the avoided crossings suggests that the spin ensembles of these isolated phosphorus donors couple strongly to the LER. We determine the corresponding coupling rate $g_{\text {eff }} / 2 \pi=1.54 \mathrm{MHz}$ from the vacuum Rabi splitting at $B_{0}=170.19 \mathrm{mT}$, corresponding to a cooperativity $C=g_{\text {eff }}^{2} /\left(\kappa_{c} \gamma_{s}\right)=12.2$. Note that the single spinresonator coupling rate is not spatially uniform [25,59].

We obtain information about further spin species present in the sample by analyzing the resonator linewidth $\kappa_{c}$ as a function of the magnetic field outside the avoided crossings from the data in Fig. 1(e). We find in Fig. 1(f) a broad structure at $B_{0}=171.5 \mathrm{mT}$, which is assigned to dangling bond defects at the $(100) \mathrm{Si} / \mathrm{SiO}_{2}$ interface, also known as $\mathrm{P}_{\mathrm{b} 0} / \mathrm{P}_{\mathrm{b} 1}$ defects $[60,61]$, and a sharp signature at $B_{0}=$ $172.2 \mathrm{mT}$ corresponding to statistically formed exchangecoupled donor pairs, called $\mathrm{P}_{2}$ dimers, with a concentration $\left[\mathrm{P}_{2}\right] \ll[\mathrm{P}][62-65]$. The analysis of this $\mathrm{P}_{2}$ dimer peak [66] yields a spin relaxation rate $\gamma_{s, \mathrm{P}_{2}} / 2 \pi=1.74 \mathrm{MHz}$ and an effective coupling rate $g_{\text {eff, } \mathrm{P}_{2}} / 2 \pi=0.35 \mathrm{MHz}$. This sets the $\mathrm{P}_{2}$ dimers in the weak coupling regime with $C=0.13$, as expected from the $\sqrt{N}$ scaling of $g_{\text {eff }}[14,67]$. Hence, we can use these two spin ensembles to directly compare the dynamics in the weak and strong coupling regime under the same experimental conditions.

Pulsed ESR spectroscopy.-In a next step, we now apply a Hahn-type echo sequence based on two Gaussian-shaped pulses with a width of 1 and $2 \mu \mathrm{s}$ and a pulse spacing of $\tau=80 \mu$ s. We use a fixed frequency $\omega_{p} / 2 \pi=4.8116 \mathrm{GHz}$, even though $\omega_{c}$ slightly shifts with $B_{0}$ [see Fig. 1(e)]. Figure 2(a) shows the Hahn echo-detected field sweep of the first echo in the time domain, where we have set the origin of the time axis to the maximum of this first echo. Note that all data shown here are single-shot measurements and no signal averaging is performed. The time interval between measurements at subsequent field points is $300 \mathrm{~s}$, chosen to be long compared to the spin relaxation time $T_{1}=(32.4 \pm 0.8) \mathrm{s}$ (see Supplemental Material [25]). From an analysis of the collective coupling rate, we estimate the absolute number of spins addressed in the spin echo to be $\approx 1.06 \times 10^{10}$ [25]. For the spin sensitivity, we obtain $\approx 1.15 \times 10^{5}$ spins $/ \sqrt{\mathrm{Hz}}$ assuming a repetition time of $5 T_{1}$ and a signal-to-noise ratio of $1[18,19]$.

Figure 2(b) displays the echo area $A_{\text {echo }}=\int_{\text {echo }}\left|S_{21}\right| d t$ using the data from Fig. 2(a) showing three peaks corresponding to the hyperfine as well as the $\mathrm{P}_{2}$ dimer transition. Evidently, the linewidths of the hyperfine transitions are much wider than expected from $\gamma_{s}$. Because of the presence of strong coupling, the spin system hybridizes such that the linewidth should also reflect $g_{\text {eff }}$. Additionally, both peaks are asymmetric with a tail toward larger magnetic fields, which we attribute to the excitation with a fixed $\omega_{p}$ without compensating for the dispersion of the avoided crossing. 
(a)

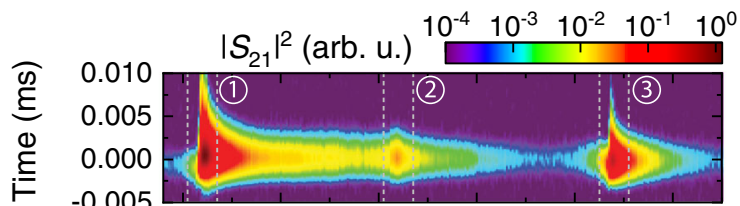

(b)

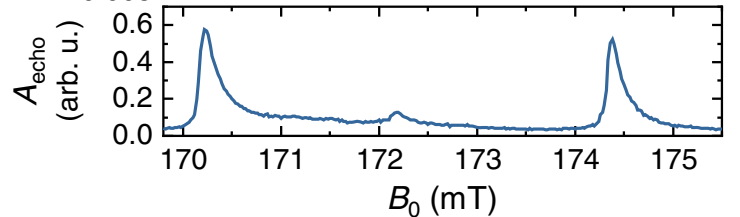

(c)
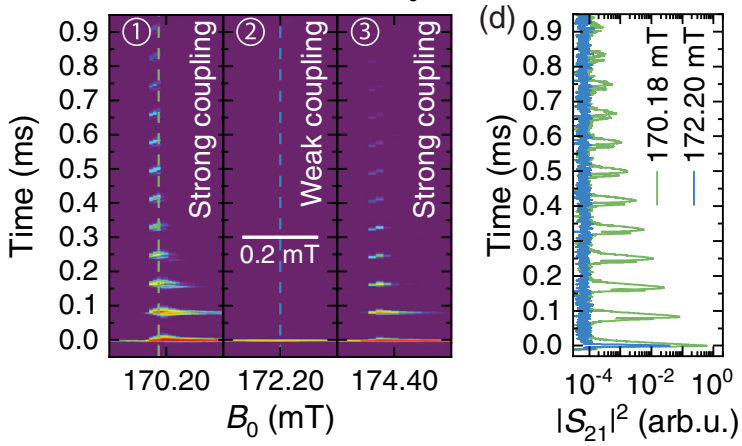

(e)



FIG. 2. (a) Echo signal as a function of acquisition time and magnetic field of the first echo. Dashed lines indicate the subpanels in (c). (b) Integrated echo area $A_{\text {echo }}=\int_{\text {echo }}\left|S_{21}\right| d t$ as a function of magnetic field, showing the two hyperfine transitions as well as the $\mathrm{P}_{2}$ dimer peak. (c) Microwave signal intensity $\left|S_{21}\right|^{2}$ displaying several echo signals after the conventional first echo for the strongly coupled hyperfine lines [1 and (3), while only one echo is visible for weakly coupled $\mathrm{P}_{2}$ dimers [2]. (d) Microwave signal intensity $\left|S_{21}\right|^{2}$ for fixed magnetic field [cf. dashed lines in (c)] for the hyperfine transition (green) and $\mathrm{P}_{2}$ line (blue). (e) Temporal evolution of the average resonator photon number $|\langle a\rangle|^{2} / N$ (upper), the average spin expectation values $S_{\mathrm{av}}^{x, y}=\sum_{j}\left\langle\sigma_{j}^{x, y}\right\rangle / N$ (middle), and $S_{\mathrm{av}}^{z}=$ $\sum_{j}\left\langle\sigma_{j}^{z}\right\rangle / N$ (lower) calculated from the semiclassical MaxwellBloch equations. The inset shows an enlargement of the gray shaded area.

Moreover, the absence of a clear echo corresponding to the $\mathrm{P}_{\mathrm{b} 0} / \mathrm{P}_{\mathrm{b} 1}$ defects is related to their small $T_{2}=22 \mu \mathrm{s}$ [25].

The conclusion we draw from this analysis is that one observes the first conventional Hahn echo (at $t=0$ ) for both the weakly and the strongly coupled spin ensembles.
The fundamental difference between weak and strong coupling manifests itself only on longer timescales, as shown in Fig. 2(c). Subpanels (1) and (3), corresponding to the strong coupling case, deserve particular attention, corresponding to the strong coupling case. Here, the first Hahn echo is followed by a periodic sequence of echo signatures, which are timed with a delay equal to the pulse delay $\tau$. In contrast, only the first conventional Hahn echo is present for the weakly coupled $\mathrm{P}_{2}$ dimers shown in subpanel (2) [68].

This marked difference is even more apparent in Fig. 2(d), where we show time traces recorded at the fixed magnetic fields of $B_{0}=172.20$ and $170.18 \mathrm{mT}$ [dashed lines in Fig. 2(c)] corresponding to the weak and strong coupling regime. While only the first conventional Hahn echo appears for the $\mathrm{P}_{2}$ dimers $\left[T_{2, \mathrm{P}_{2}}=(4.67 \pm 0.13) \mathrm{ms}\right.$; Supplemental Material [25] ], we observe 12 echoes separated by $\tau$ for the strongly coupled hyperfine transitions $\left[T_{2, \mathrm{P}}=(2.37 \pm 0.08) \mathrm{ms}[25]\right]$. The echo signatures in the echo train exhibit an underlying substructure going beyond the scope of this Letter. Although several mechanisms of multiple echo generation are known in the literature, the absence of multiple echoes for the $\mathrm{P}_{2}$ dimers excludes these for a possible explanation (see also the Supplemental Material [25] for more details). This suggests that the detection of the multiple echoes is, indeed, related to the strong coupling regime.

The relevant mechanism leading to this unique dynamical evolution can be best understood when revisiting the conventional Hahn echo sequence shown in Fig. 1(a). For simplicity, we assume here that all spins end up in the $x y$ plane after the first $\pi / 2$ pulse (see panels $1-3$ ), although the spatial variation of the excitation field $B_{1}$ and the frequency distribution of the spin ensemble inevitably lead to rotation errors. Realistically, the net dipole moment generated in the $x y$ plane during this first pulse leads to a strong collective coupling with the resonator and, hence, rapid deexcitation of the spin system. However, dephasing quickly reduces this dipole moment and thereby effectively suppresses this spin decay channel. After an evolution time $\tau$, the second pulse is injected to start the refocusing process. A perfect $\pi$ pulse would lead to a refocusing of all spins after another time span $\tau$, creating the first (conventional) Hahn echo without any subsequent echoes. With the rotation angle realistically deviating from $\pi$, however, the refocusing is imperfect and the spins end up at different latitudes on the Bloch sphere, depending on their detuning $\delta \omega$ from the average Larmor frequency (see panels 3-5). This mechanism can also be understood as a frequency encoding of spin packets depending on their orientation on the Bloch sphere at the arrival time of this imperfect $\pi$ pulse. Specifically, we identify spin packets that point in opposite directions on the $S_{y}$ axis when the imperfect $\pi$ pulse arrives using red and blue arrows in the panels in Fig. 1(a). These will be particularly relevant for the subsequent pulse train. 
Their frequency detunings are determined by those multiples of $\pi$ rotations that the spins already undertook at the arrival of the refocusing pulse: $\delta \omega=2 n \pi / \tau$ (red spins) and $\delta \omega=(2 n+1) \pi / \tau$ (blue spins) with $n \in \mathbb{Z}$. In this way, spins with significantly different individual detuning values $\delta \omega$ are now encoded in the same packet. At a time $\tau$ after the imperfect $\pi$ pulse, when spins (partially) refocus, they emit the first (conventional) Hahn echo through the coupling to the resonator. Notably, the net dipole moment in the $x y$ plane created in this refocusing process, together with the strong coupling to the microwave field, also leads to a significant spin decay. Importantly, this decay is realized on the Bloch sphere as a spin rotation during this first Hahn echo that affects the projection of the dipole moment on the $x y$ plane differently for the blue and red spin packets (see panels 5 and 6). This rotation becomes significant at a time $\tau$ after the first Hahn echo, where these spin packets again point in opposite $S_{y}$ directions (red and blue arrows in panel 7): with the $x y$ projection of these two spin vectors now having different lengths, they give rise to another net dipole moment that produces the (unconventional) second Hahn echo. Here, the process starts all over again, producing the third echo, etc.

Note that, without the spin rotation during the first Hahn echo, the red and blue spins would maintain the same $x y$ projection, such that no net dipole is created and therefore also no subsequent echoes. In this way, one not only understands how the generation of one echo gives rise to the next one, but also why the strong coupling regime is essential: for weak coupling also the spin rotation by deexcitation through the resonator is weak, such that all unconventional echoes are negligibly small. Moreover, also imperfect rotation angles are essential (as induced, e.g., by the inhomogeneities in the system), as no frequency encoding of spin packets would occur otherwise (for more information, see the Supplemental Material [25]).

Theoretical description.-To underpin this heuristic explanation, we set up a theoretical model based on the inhomogeneous Tavis-Cummings Hamiltonian,

$$
\begin{aligned}
\mathcal{H}= & \hbar \Delta_{c} a^{\dagger} a+\frac{\hbar}{2} \sum_{j=1}^{N} \Delta_{j} \sigma_{j}^{z}+\sum_{j=1}^{N} \hbar\left[g_{j} \sigma_{j}^{-} a^{\dagger}+g_{j}^{*} \sigma_{j}^{+} a\right] \\
& +i \hbar\left[\eta(t) a^{\dagger}-\eta^{*}(t) a\right],
\end{aligned}
$$

where $\Delta_{c} \equiv \omega_{c}-\omega_{p}$ and $\Delta_{j} \equiv \omega_{j}-\omega_{p}$ are the detunings of the resonator frequency $\omega_{c}$ and of the individual spin frequencies $\omega_{j}$ from the carrier frequency $\omega_{p}$ of the incoming microwave pulse with amplitude $\eta(t)$. Here, $a^{\dagger}$ $(a)$ is the creation (annihilation) operator for the resonator mode coupling with $g_{j}$ to the $j$ th spin, which is described by the standard Pauli operators $\sigma_{j}^{z}, \sigma_{j}^{+}$, and $\sigma_{j}^{-}$. Note that (1) does not include direct dipole-dipole interactions, whichalthough present in the actual sample-do not seem to play a fundamental role for the formation of the echo pulses in our model. For large spin ensembles, we can use a mean-field formulation in the form of the MaxwellBloch equations for the resonator and spin expectation values $[25,69]$,

$$
\begin{gathered}
\frac{d}{d t}\langle a\rangle=-\left[\kappa_{c}+i\left(\Delta_{c}\right)\right]\langle a\rangle-i \sum_{j=1}^{N} g_{j}\left\langle\sigma_{j}^{-}\right\rangle+\eta(t), \\
\frac{d}{d t}\left\langle\sigma_{j}^{-}\right\rangle=-\left[\gamma_{\perp}+i\left(\Delta_{j}\right)\right]\left\langle\sigma_{j}^{-}\right\rangle+i g_{j}\left\langle\sigma_{j}^{z}\right\rangle\langle a\rangle, \\
\frac{d}{d t}\left\langle\sigma_{j}^{z}\right\rangle=-\gamma_{\|}\left(\left\langle\sigma_{j}^{z}\right\rangle+1\right)+2 i g_{j}\left(\left\langle\sigma_{j}^{-}\right\rangle\left\langle a^{\dagger}\right\rangle-\text { c.c. }\right) .
\end{gathered}
$$

Here, $\gamma_{\perp}=1 / T_{2}\left(\gamma_{\|}=1 / T_{1}\right)$ is the transverse (longitudinal) spin relaxation rate. We account for the dephasing of the spin ensemble by introducing the phenomenological Lorentzian spin spectral density, $\rho(\omega)=\left\{\pi \gamma_{s}[1+\right.$ $\left.\left.\left(\omega-\omega_{s}\right)^{2} / \gamma_{s}^{2}\right]\right\}^{-1}$, with width $\gamma_{s}$ and mean frequency $\omega_{s}$, characterizing the frequency distribution of the spin ensemble $[23,25,70]$. For simplicity, we assume for the calculations presented in Fig. 2(e) that all spins couple with the mean coupling strength $g_{j}=g_{0}=g_{\text {eff }} / \sqrt{N}$ (in the Supplemental Material [25], we discuss the impact of a distribution of $g_{j}$ ).

To calculate the dynamics of the spin-resonator system, we numerically solve the Maxwell-Bloch equations (2)-(4) for two rectangular driving pulses with a width of 1 and $2 \mu \mathrm{s}$, a pulse delay of $\tau=80 \mu \mathrm{s}$, and a pulse amplitude of $\eta / \kappa_{c}=1.08 \times 10^{5}$. Furthermore, we set $\omega_{c}=\omega_{p}$, while the mean frequency of the spin ensemble is slightly detuned from the resonator frequency by $\left(\omega_{s}-\omega_{c}\right) / 2 \pi=$ $0.14 \mathrm{MHz}$ to match the experimental conditions in the strong coupling regime (at $B_{0}=170.18 \mathrm{mT}$ ).

The calculated average resonator photon number $|\langle a(t)\rangle|^{2} / N$ following an ordinary Hahn echo sequence is presented in the upper panel of Fig. 2(e). Most importantly, we find that these numerical results nicely reproduce the multiple echo signatures found experimentally [see Fig. 2(d)], using only minimalistic assumptions. Additionally, these simulations provide the average spin expectation values $S_{\mathrm{av}}^{x, y, z}:=\sum_{j}\left\langle\sigma_{j}^{x, y, z}\right\rangle / N$, which are not directly accessible in the experiment. From these quantities, we can directly evaluate the macroscopic dipole moment $\sum_{j}\left\langle\sigma_{j}^{-}\right\rangle=N\left(S_{\mathrm{av}}^{x}+i S_{\mathrm{av}}^{y}\right)$, which couples the spin dynamics to the resonator field via (2). Hereby, we can directly confirm, e.g., that the arrival of the first conventional Hahn echo, at $t=0$, is accompanied by peaks in the average dipole moments $S_{\mathrm{av}}^{x}$ and $S_{\mathrm{av}}^{y}$, leading to a resonatorenhanced decay of the spin excitation $S_{\mathrm{av}}^{z}$ [see also gray inset of Fig. 2(e)]. Confirming our heuristic model from above, the same coincidence between peaks in the dipole moments of $S_{\mathrm{av}}^{x}, S_{\mathrm{av}}^{y}$, the steps in the decay of $S_{\mathrm{av}}^{z}$, and the emission of a photon pulse into the resonator is observed 
for all subsequent (unconventional) Hahn echoes. This reduced model thus already reproduces all salient features of the experiment. As shown explicitly in the Supplemental Material [25], the spin rotations on the Bloch sphere occurring during the emission of a Hahn echo are essential to produce the subsequent echo, a feature which is connected to strong spin-resonator coupling. We also checked in [25] that imperfections in the second applied $(\pi)$ pulse are required for the observation of multiple echoes. Next steps in the improvement of the model shall include the dipole-dipole interactions between the spins, as well as the inclusion of the exact shape of the spectral spin and spatial coupling distributions.

In conclusion, we compared continuous-wave and pulsed ESR measurements on a weakly and strongly coupled spin ensemble using superconducting lumped element resonators. We observed a self-sustained train of periodic echo signatures after applying a Hahn echo sequence to the spin ensemble in the strong coupling regime and explain this effect using a simple model based on the inhomogeneous Tavis-Cummings Hamiltonian. Our Letter establishes a robust and self-sustained dynamical phenomenon in strongly coupled hybrid spin-photon systems, which may be relevant for quantum memory protocols.

We acknowledge stimulating discussions with K. Lips, S. T. B. Goennenwein, D. Einzel, and M. Weiler and financial support from the Deutsche Forschungsgesellschaft via Germany's Excellence Strategy EXC-2111-390814868 and SPP 1601 (HU 1896/2-1). M.Z. and S. R. acknowledge support by the European Commission under Project NHQWAVE No. MSCA-RISE 691209 and by the Austrian Science Fund (FWF) through the Doctoral Program CoQuS (W1210). M. Z. would like to thank the Institute for Theoretical Atomic, Molecular, and Optical Physics (ITAMP) at Harvard for hospitality.

Note added.-Recently, we became aware of a related work by Debnath et al. [71].

*Present address: London Centre for Nanotechnology, University College London, London WC1H $0 \mathrm{AH}$, United Kingdom.

†hans.huebl@wmi.badw.de

[1] T. Prisner, M. Rohrer, and F. MacMillan, Pulsed EPR spectroscopy: Biological applications, Annu. Rev. Phys. Chem. 52, 279 (2001).

[2] S.S. Eaton and G. R. Eaton, Multifrequency pulsed EPR and the characterization of molecular dynamics, in Methods in Enzymology (Elsevier, New York, 2015), Vol. 563, p. 37.

[3] P. G. Baranov, H. J. von Bardeleben, F. Jelezko, and J. Wrachtrup, Magnetic Resonance of Semiconductors and Their Nanostructures, Springer Series in Materials Science Vol. 253 (Springer, Vienna, 2017).
[4] R. Schirhagl, K. Chang, M. Loretz, and C. L. Degen, Nitrogen-vacancy centers in diamond: Nanoscale sensors for physics and biology, Annu. Rev. Phys. Chem. 65, 83 (2014).

[5] M.H. Devoret and R. J. Schoelkopf, Superconducting circuits for quantum information: An outlook, Science 339, 1169 (2013).

[6] F. A. Zwanenburg, A. S. Dzurak, A. Morello, M. Y. Simmons, L. C. L. Hollenberg, G. Klimeck, S. Rogge, S. N. Coppersmith, and M. A. Eriksson, Silicon quantum electronics, Rev. Mod. Phys. 85, 961 (2013).

[7] A. Schweiger and G. Jeschke, Principles of Pulse Electron Paramagnetic Resonance (Oxford University Press, Oxford, England, New York, 2001).

[8] E. L. Hahn, Spin echoes, Phys. Rev. 80, 580 (1950).

[9] Y. Kubo, F. R. Ong, P. Bertet, D. Vion, V. Jacques, D. Zheng, A. Dréau, J.-F. Roch, A. Auffeves, F. Jelezko, J. Wrachtrup, M. F. Barthe, P. Bergonzo, and D. Esteve, Strong Coupling of a Spin Ensemble to a Superconducting Resonator, Phys. Rev. Lett. 105, 140502 (2010).

[10] D. I. Schuster, A. P. Sears, E. Ginossar, L. DiCarlo, L. Frunzio, J. J. L. Morton, H. Wu, G. A. D. Briggs, B. B. Buckley, D. D. Awschalom, and R. J. Schoelkopf, HighCooperativity Coupling of Electron-Spin Ensembles to Superconducting Cavities, Phys. Rev. Lett. 105, 140501 (2010).

[11] R. Amsüss, C. Koller, T. Nöbauer, S. Putz, S. Rotter, K. Sandner, S. Schneider, M. Schramböck, G. Steinhauser, H. Ritsch, J. Schmiedmayer, and J. Majer, Cavity QED with Magnetically Coupled Collective Spin States, Phys. Rev. Lett. 107, 060502 (2011).

[12] S. Probst, H. Rotzinger, S. Wünsch, P. Jung, M. Jerger, M. Siegel, A. V. Ustinov, and P. A. Bushev, Anisotropic Rare-Earth Spin Ensemble Strongly Coupled to a Superconducting Resonator, Phys. Rev. Lett. 110, 157001 (2013).

[13] S. Putz, D. O. Krimer, R. Amsüss, A. Valookaran, T. Nöbauer, J. Schmiedmayer, S. Rotter, and J. Majer, Protecting a spin ensemble against decoherence in the strong-coupling regime of cavity QED, Nat. Phys. 10, 720 (2014).

[14] C. W. Zollitsch, K. Mueller, D. P. Franke, S. T. B. Goennenwein, M. S. Brandt, R. Gross, and H. Huebl, High cooperativity coupling between a phosphorus donor spin ensemble and a superconducting microwave resonator, Appl. Phys. Lett. 107, 142105 (2015).

[15] J. J. L. Morton, A. M. Tyryshkin, R. M. Brown, S. Shankar, B. W. Lovett, A. Ardavan, T. Schenkel, E. E. Haller, J. W. Ager, and S. A. Lyon, Solid-state quantum memory using the ${ }^{31} \mathrm{P}$ nuclear spin, Nature (London) 455, 1085 (2008).

[16] P. Bushev, A. K. Feofanov, H. Rotzinger, I. Protopopov, J. H. Cole, C. M. Wilson, G. Fischer, A. Lukashenko, and A. V. Ustinov, Ultralow-power spectroscopy of a rare-earth spin ensemble using a superconducting resonator, Phys. Rev. B 84, 060501(R) (2011).

[17] C. Grezes, Y. Kubo, B. Julsgaard, T. Umeda, J. Isoya, H. Sumiya, H. Abe, S. Onoda, T. Ohshima, K. Nakamura, I. Diniz, A. Auffeves, V. Jacques, J.-F. Roch, D. Vion, D. Esteve, K. Moelmer, and P. Bertet, Towards a spin-ensemble quantum memory for superconducting qubits, C. R. Phys. 17, 693 (2016). 
[18] A. Bienfait, J. J. Pla, Y. Kubo, M. Stern, X. Zhou, C. C. Lo, C. D. Weis, T. Schenkel, M. L. W. Thewalt, D. Vion, D. Esteve, B. Julsgaard, K. Mølmer, J. J. L. Morton, and P. Bertet, Reaching the quantum limit of sensitivity in electron spin resonance, Nat. Nanotechnol. 11, 253 (2016).

[19] C. Eichler, A. J. Sigillito, S. A. Lyon, and J. R. Petta, Electron Spin Resonance at the Level of $10^{4}$ Spins Using Low Impedance Superconducting Resonators, Phys. Rev. Lett. 118, 037701 (2017).

[20] M. H. Levitt, Spin Dynamics: Basics of Nuclear Magnetic Resonance, 2nd ed. (John Wiley \& Sons, Hoboken, NJ, 2008).

[21] B. C. Rose, A. M. Tyryshkin, H. Riemann, N. V. Abrosimov, P. Becker, H.-J. Pohl, M. L.W. Thewalt, K. M. Itoh, and S. A. Lyon, Coherent Rabi Dynamics of a Superradiant Spin Ensemble in a Microwave Cavity, Phys. Rev. X 7, 031002 (2017).

[22] S. Putz, A. Angerer, D. O. Krimer, R. Glattauer, W. J. Munro, S. Rotter, J. Schmiedmayer, and J. Majer, Spectral hole burning and its application in microwave photonics, Nat. Photonics 11, 36 (2017).

[23] A. Angerer, S. Putz, D. O. Krimer, T. Astner, M. Zens, R. Glattauer, K. Streltsov, W. J. Munro, K. Nemoto, S. Rotter, J. Schmiedmayer, and J. Majer, Ultralong relaxation times in bistable hybrid quantum systems, Sci. Adv. 3, e1701626 (2017).

[24] J. P. Gordon and K. D. Bowers, Microwave Spin Echoes from Donor Electrons in Silicon, Phys. Rev. Lett. 1, 368 (1958).

[25] See Supplemental Material at http://link.aps.org/ supplemental/10.1103/PhysRevLett.125.137701 for details on the experimental setup and the theoretical description, as well as additional measurements, including spin life time and spin coherence time measurements, which includes Refs. [26-57].

[26] CST Microwave Studio 2016, CST Computer Simulation Technology, GmbH (2016), https://www.cst.com.

[27] J. H. Wesenberg, A. Ardavan, G. A. D. Briggs, J. J. L. Morton, R. J. Schoelkopf, D. I. Schuster, and K. Mølmer, Quantum Computing with an Electron Spin Ensemble, Phys. Rev. Lett. 103, 070502 (2009).

[28] G. Feher, Electron spin resonance experiments on donors in silicon. I. Electronic structure of donors by the electron nuclear double resonance technique, Phys. Rev. 114, 1219 (1959).

[29] R. J. Schoelkopf and S. M. Girvin, Wiring up quantum systems, Nature (London) 451, 664 (2008).

[30] I. Chiorescu, N. Groll, S. Bertaina, T. Mori, and S. Myiashita, Magnetic strong coupling in a spin-photon system and transition to classical regime, Phys. Rev. B 82, 024413 (2010).

[31] A. Bienfait, J. J. Pla, Y. Kubo, X. Zhou, M. Stern, C. C. Lo, C. D. Weis, T. Schenkel, D. Vion, D. Esteve, J. J. L. Morton, and P. Bertet, Controlling spin relaxation with a cavity, Nature (London) 531, 74 (2016).

[32] J. R. Klauder and P. W. Anderson, Spectral diffusion decay in spin resonance experiments, Phys. Rev. 125, 912 (1962).

[33] A. M. Tyryshkin, S. A. Lyon, A. V. Astashkin, and A. M. Raitsimring, Electron spin relaxation times of phosphorus donors in silicon, Phys. Rev. B 68, 193207 (2003).
[34] K. M. Salikhov, S. A. Dzuba, and A. M. Raitsimring, The theory of electron spin-echo signal decay resulting from dipole-dipole interactions between paramagnetic centers in solids, J. Magn. Reson. 42, 255 (1981).

[35] A. M. Tyryshkin, S. Tojo, J. J. L. Morton, H. Riemann, N. V. Abrosimov, P. Becker, H.-J. Pohl, T. Schenkel, M. L. W. Thewalt, K. M. Itoh, and S. A. Lyon, Electron spin coherence exceeding seconds in high-purity silicon, Nat. Mater. 11, 143 (2012).

[36] D. R. Taylor, J. R. Marko, and I. G. Bartlet, Exchange and dipolar fields in phosphorus-doped silicon measured by electron spin resonance echoes, Solid State Commun. 14, 295 (1974).

[37] M. H. Alaimo and J.E. Roberts, Effects of paramagnetic cations on the nonexponential spin-lattice relaxation of rare spin nuclei in solids, Solid State Nucl. Magn. Reson. 8, 241 (1997).

[38] N. Bloembergen, On the interaction of nuclear spins in a crystalline lattice, Physica (Utrecht) 15, 386 (1949).

[39] K. W. Eberhardt, S. Mouaziz, G. Boero, J. Brugger, and B. H. Meier, Direct Observation of Nuclear Spin Diffusion in Real Space, Phys. Rev. Lett. 99, 227603 (2007).

[40] A. G. Redfield, Spatial diffusion of spin energy, Phys. Rev. 116, 315 (1959).

[41] B.E. Vugmeister, Spatial and spectral spin diffusion in dilute spin systems, Phys. Status Solidi B 76, 161 (1976).

[42] G. Feher and E. A. Gere, Electron spin resonance experiments on donors in silicon. II. Electron spin relaxation effects, Phys. Rev. 114, 1245 (1959).

[43] A. Morello, J. J. Pla, F. A. Zwanenburg, K. W. Chan, K. Y. Tan, H. Huebl, M. Mottonen, C. D. Nugroho, C. Yang, J. A. van Donkelaar, A. D. C. Alves, D. N. Jamieson, C. C. Escott, L. C. L. Hollenberg, R. G. Clark, and A. S. Dzurak, Single-shot readout of an electron spin in silicon, Nature (London) 467, 687 (2010).

[44] H. Hasegawa, Spin-lattice relaxation of shallow donor states in $\mathrm{Ge}$ and $\mathrm{Si}$ through a direct phonon process, Phys. Rev. 118, 1523 (1960).

[45] M. Tavis and F. W. Cummings, Exact solution for an Nmolecule-radiation-field Hamiltonian, Phys. Rev. 170, 379 (1968).

[46] H. J. Carmichael, Statistical Methods in Quantum Optics 2: Non-Classical Fields, Theoretical and Mathematical Physics (Springer, Berlin, Heidelberg, 2007).

[47] K. Sandner, H. Ritsch, R. Amsüss, Ch. Koller, T. Nöbauer, S. Putz, J. Schmiedmayer, and J. Majer, Strong magnetic coupling of an inhomogeneous nitrogen-vacancy ensemble to a cavity, Phys. Rev. A 85, 053806 (2012).

[48] G. Deville, M. Bernier, and J. M. Delrieux, NMR multiple echoes observed in solid ${ }^{3} \mathrm{He}$, Phys. Rev. B 19, 5666 (1979).

[49] G. Eska, H.-G. Willers, B. Amend, and W. Wiedemann, Spin echo experiments in superfluid 3He, Physica (Amsterdam) 108B+C, 1155 (1981).

[50] D Einzel, G Eska, Y Hirayoshi, T Kopp, and P Wolfle, Multiple Spin Echoes in a Normal Fermi Liquid, Phys. Rev. Lett. 53, 2312 (1984).

[51] R. Bowtell, R. M. Bowley, and P. Glover, Multiple spin echoes in liquids in a high magnetic field, J. Magn. Reson. 88, 643 (1990). 
[52] A. S. Bedford, R. M. Bowley, J. R. Owers-Bradley, and D. Wightman, Multiple spin echoes in spin polarized Fermi liquids, J. Low Temp. Phys. 85, 389 (1991).

[53] R. Bowtell and P. Robyr, Structural Investigations with the Dipolar Demagnetizing Field in Solution NMR, Phys. Rev. Lett. 76, 4971 (1996).

[54] W. S. Warren, S. Lee, W. Richter, and S. Vathyam, Correcting the classical dipolar demagnetizing field in solution NMR, Chem. Phys. Lett. 247, 207 (1995).

[55] S. Bloom, Effects of radiation damping on spin dynamics, J. Appl. Phys. 28, 800 (1957).

[56] M. P. Augustine, Transient properties of radiation damping, Prog. Nucl. Magn. Reson. Spectrosc. 40, 111 (2002).

[57] A. Vlassenbroek, J. Jeener, and P. Broekaert, Radiation damping in high resolution liquid NMR: A simulation study, J. Chem. Phys. 103, 5886 (1995).

[58] S. Probst, F. B. Song, P. A. Bushev, A. V. Ustinov, and M. Weides, Efficient and robust analysis of complex scattering data under noise in microwave resonators, Rev. Sci. Instrum. 86, 024706 (2015).

[59] S. Weichselbaumer, P. Natzkin, C. W. Zollitsch, M. Weiler, R. Gross, and H. Huebl, Quantitative Modeling of Superconducting Planar Resonators for Electron Spin Resonance, Phys. Rev. Applied 12, 024021 (2019).

[60] E. H. Poindexter, P. J. Caplan, B. E. Deal, and R. R. Razouk, Interface states and electron spin resonance centers in thermally oxidized (111) and (100) silicon wafers, J. Appl. Phys. 52, 879 (1981).

[61] A. Stesmans and V. V. Afanas'ev, Electron spin resonance features of interface defects in thermal (100) $\mathrm{Si} / \mathrm{SiO}_{2}$, J. Appl. Phys. 83, 2449 (1998).

[62] G. Feher, R. C. Fletcher, and E. A. Gere, Exchange effects in spin resonance of impurity atoms in silicon, Phys. Rev. 100, 1784 (1955).
[63] D. Jérome and J. M. Winter, Electron spin resonance on interacting donors in silicon, Phys. Rev. 134, A1001 (1964).

[64] K. Morigaki and S. Maekawa, Electron spin resonance studies of interacting donor clusters in phosphorus-doped silicon, J. Phys. Soc. Jpn. 32, 462 (1972).

[65] S. Shankar, A. M. Tyryshkin, and S. A. Lyon, ESR measurements of phosphorus dimers in isotopically enriched ${ }^{28}$ si silicon, Phys. Rev. B 91, 245206 (2015).

[66] P. F. Herskind, A. Dantan, J. P. Marler, M. Albert, and M. Drewsen, Realization of collective strong coupling with ion Coulomb crystals in an optical cavity, Nat. Phys. 5, 494 (2009).

[67] H. Huebl, C. W. Zollitsch, J. Lotze, F. Hocke, M. Greifenstein, A. Marx, R. Gross, and S. T. B. Goennenwein, High Cooperativity in Coupled Microwave Resonator Ferrimagnetic Insulator Hybrids, Phys. Rev. Lett. 111, 127003 (2013).

[68] Note that we use for the $\mathrm{P}_{2}$ dimer measurements the same measurement protocol as for hyperfine transitions. Within the noise budget, a second echo should be detectable, if its echo amplitudes scale in the same manner as for the strong coupling case. However, we do not observe such a subsequent echo.

[69] M. Zens, D. O. Krimer, and S. Rotter, Critical phenomena and nonlinear dynamics in a spin ensemble strongly coupled to a cavity. II. Semiclassical-to-quantum boundary, Phys. Rev. A 100, 013856 (2019).

[70] D. O. Krimer, M. Zens, and S. Rotter, Critical phenomena and nonlinear dynamics in a spin ensemble strongly coupled to a cavity. I. Semiclassical approach, Phys. Rev. A 100, 013855 (2019).

[71] K. Debnath, G. Dold, J. J. L. Morton, and K. Mølmer, following Letter, Self-Stimulated Pulse Echo Trains from Inhomogeneously Broadened Spin Ensembles, Phys. Rev. Lett. 125, 137702 (2020). 